\title{
Clinical Differences Between Nonarteritic Anterior Ischemic Optic Neuropathy and Optic Neuritis in Chinese Patients: A Retrospective Comparative Case Series Study
}

\author{
Jun Chen ${ }^{1,2}$, Wanghua Zhao ${ }^{1}$, Zacharia Ackbarkhan ${ }^{1}$, Jianfeng He ${ }^{1}$, Yi Du ${ }^{1, ~ * ~}$ \\ ${ }^{1}$ Department of Ophthalmology, First Affiliated Hospital of Guangxi Medical University, Nanning, China \\ ${ }^{2}$ Department of Ophthalmology, Affiliated Nanping First Hospital of Fujian Medical University, Nanping, China \\ Email address: \\ 329994568@qq.com (Jun Chen),673614962@qq.com(Wanghua Zhao),3564256483@qq.com (Z. Ackbarkhan), \\ hejianf@foxmail.com (Jianfeng He), duyi@gxmu.edu.cn (Yi Du) \\ ${ }^{*}$ Corresponding author
}

\section{To cite this article:}

Jun Chen, Wanghua Zhao, Zacharia Ackbarkhan, Jianfeng He, Yi Du. Clinical Differences Between Nonarteritic Anterior Ischemic Optic Neuropathy and Optic Neuritis in Chinese Patients: A Retrospective Comparative Case Series Study. International Journal of Ophthalmology \& Visual Science. Vol. 4, No. 1, 2019, pp. 7-13. doi: 10.11648/j.ijovs.20190401.12

Received: January 6, 2019; Accepted: January 30, 2019; Published: February 25, 2019

\begin{abstract}
The aim of this study is to compare the clinical features between nonarteritic anterior ischemic optic neuropathy (NAION) and optic neuritis (ON) in Chinese patients. The clinical features of 23 cases ( 23 eyes) of NAION and 62 cases ( 80 eyes) of ON, including their general conditions, manifestations, auxiliary examinations were retrospectively compared. Simultaneous onset of bilateral eye $(29.03 \%$ vs. $0 \%, \mathrm{p}=0.009)$ and pain on movement of the eyes $(23.75 \%$ vs. $0 \%, \mathrm{p}=0.022)$ were more common in ON patients in comparison to NAION patients. Ocular pain $(32.5 \%$ vs. $4.35 \%, \mathrm{p}=0.007)$ was higher in ON patients than that in NAION patients. Optic disc edema $(100 \%$ vs. $62.5 \%, \mathrm{p}<0.001)$, peripapillary hemorrhage $(47.83 \%$ vs. $10 \%, \mathrm{p}<0.001)$ and peripapillary hard exudates $(17.39 \%$ vs. $1.25 \%, \mathrm{p}=0.009)$ in NAION patients were more common compared to ON patients,. During fluorescein angiography, the rates of the partial or whole papillary hypofluorescent in early phase $(65.21 \%$ vs. $22.86 \%$, $\mathrm{p}<0.001)$, peripapillary choroidal filling delay $(73.91 \%$ vs. $14.29 \%, \mathrm{p}<0.001)$ and partial papillary hyperfluorescent in later phase $(52.17 \%$ vs. $25.71 \%, p=0.019)$ were higher in NAION patients than those in ON patients. The clinical features of NAION and $\mathrm{ON}$ in Chinese patients reveal a surprising overlap and they still have some characteristic clinical features that can be utilized to differentiate the two diseases.
\end{abstract}

Keywords: Nonarteritic Anterior Ischemic Optic Neuropathy, Optic Neuritis, Retrospective Study, Chinese Patients, Distinct Features

\section{Introduction}

Nonarteritic anterior ischemic optic neuropathy (NAION) and optic neuritis (ON), two of the most common neuro-ophthalmic diseases in adults, share many symptoms and signs, but with a rather different prognostic implication [1].

Their pathogenesis, treatment and prognosis are great different. ON presents with severe acute visual disturbance generally affecting young, otherwise healthy individuals. ON is caused by an autoimmune reaction directed against the optic nerve and it may be the first manifestation of multiple sclerosis [2]. NAION, on the other hand, presents with sudden painless visual loss associated with optic disc edema, resulting in visual field loss. NAION represents an acute ischemic disorder of the optic nerve head, which is supplied in blood vessels by the posterior ciliary arteries [3]. Furthermore, NAION has been historically associated with a small, crowded appearing, and cupless optic disc, termed the disc at risk [4].

Clinically, it can be difficult to differentiate between them with their initial presentation. The studies conducted in Western countries demonstrated that ocular pain is an important clue to differentiate between the two diseases [1]. However, the rates of $\mathrm{ON}$ associated with ocular pain is only $40 \%$ in China and 56\% in Japan [5, 6]. In addition, the rates of 
NAION associated with ocular pain in Western countries have been commented by several studies to be in the range of $8 \%$ to $12 \%$ [7]. There were differences in the clinical features of two diseases between Western countries and China, here, this study was set out to evaluate and analyze the clinical characteristics of ON and NAION in a Chinese population.

\section{Methods}

\subsection{Participants}

The medical records of all patients with NAION and ON were collected and reviewed, between Jan 2012 and Dec 2016, from the First Affiliated Hospital of Guangxi Medical University (Nanning), China. Inclusion criteria for enrolment included: Chinese ethnicity, age $\geq 18$ years, and onset time $\leq 4$ weeks. This retrospective study was approved by the institutional review board of the institutional review board of the First Affiliated Hospital of Guangxi Medical University. Due to the retrospective nature of the study, informed consent was waived.

\subsubsection{Inclusion Criteria}

The criteria required for diagnosis of $\mathrm{ON}$ included the following [8]: (1) Optic nerve damage correlated with visual field abnormalities; (2) A history of acute pain or painless vision loss and optic disc edema; (3) Existence of relative afferent papillary defect and/or visual evoked potential anomalies; (4) no clinical evidence of ischemic, traumatic, compressive, metabolic, hereditary or toxic optic neuropathy, or other causative ocular diseases; (5) Exclusion of diseases involving the optic chiasm, posterior to the chiasm or occipital cortex; (6) Exclusion of patients with retinopathy, maculopathy, ametropia, glaucoma or other anterior segment diseases; (7) Exclusion of nonorganic vision loss.

The criteria required for diagnosis of NAION included the following [9]: (1) A history of sudden visual loss and/or painless vision loss; (2) Visual field testing showed that quadrant field defect continuous with blind spot, most common nasal and interior, bypassed the fixation; (3) Partial or diffuse optic disc edema, generally peripapillary linear hemorrhage; (4) Relative afferent papillary defect or Visual evoked potential anomalies; (5) Present of systemic or local risk factors; (6) Exclusion of other optic neuropathy.

\subsubsection{Exclusion Criteria}

Exclusion criteria were as followed: (1) Optic neuropathy caused by inheritance, infection, glaucoma, vasculitis, compression or surgery; (2) Affirmative or suspicious arteritic anterior ischemic optic neuropathy diagnosed by biopsy pathology; (3) Unclear diagnosis or optic neuropathy which was hard to classify; (4) Patients with posterior ischemic optic neuropathy; (5) Patients who had undergone glucocorticoids before presenting our clinic; (6) Patients with any retinal or optic nerve lesions; (7) Patients with cataract, macular degeneration, intraocular pressure more than $21 \mathrm{mmHg}$ or other complications that influenced the visual acuity and visual fields; (8) Recurrent optic neuropathies; (9) Cases with incomplete data.

\subsection{Data Collection}

After eligibility was established, the data obtained from each patient included their age, gender, systemic diseases, symptoms, best corrected visual acuity, fundus examination, fluorescein angiography (FFA), visual field testing, visual evoked potentials (VEP) and blood routine examination, in order to properly differentiate between NAION and ON.

\subsection{Data Analysis}

The statistical analysis was done with the Statistical Program for the Social Sciences (SPSS, version 22.0, SPSS Inc., Chicago). Hyperlipemia and Hypertension were analyzed using Fisher's Exact test. Independent Sample T test was used to analyze age. Mann-Whitney U test was used to analyze the visual acuity (Log MAR) and VEP. Pearson Chi-Square was used to compare gender, ocular pain, optic disc color, optic disc edema, peripapillary choroidal filling delay, normal papillary fluorescence, diffuse papillary hyperfluorescent and partial papillary hyperfluorescent. Other parameters were analyzed using Correction for continuity. P Values $<0.05$ were considered as statistically significant.

\section{Results}

In this study, 62 patients ( 80 eyes) with $\mathrm{ON}$ and 23 patients (23 eyes) with NAION were enrolled.

\subsection{General Conditions Between Nonarteritic Anterior Ischemic Optic Neuropathy and Optic Neuritis Patients}

The mean age of the study participants was $53.43 \pm 9.71$ years in NAION patients and $43.74 \pm 13.41$ years in $\mathrm{ON}$ patients $(p=0.020)$. The rates of hypertension $(6.45 \%$ vs. $30.43 \%, p=0.010$ ) were lower in ON patients compared to NAION patients. Simultaneous onset of bilateral eye in ON patients $(29.03 \%$ vs. $0 \%, p=0.009$ Table 1$)$ was more common than NAION patients. The two conditions in gender, diabetes, hyperlipidemia and angiocardiopathy have no statistical difference (Table 1).

Table 1. General conditions between NAION and ON patients.

\begin{tabular}{llll}
\hline & NAION (n=23) & ON (n=62) \\
\hline Male: Female & $14: 9$ & $25: 37$ & $0.091 \dagger$ \\
Age (yr) & $53.43 \pm 9.71$ & $43.74 \pm 13.41$ & $0.020 \dagger$ \\
Hyperlipemia & $1(4.35 \%)$ & $0(0 \%)$ & $0.271 \S$ \\
Hypertension & $7(30.43 \%)$ & $4(6.45 \%)$ & $0.010 \S$ \\
Diabetes mellitus & $2(8.70 \%)$ & $0(0 \%)$ & $0.122 \|$ \\
\hline
\end{tabular}




\begin{tabular}{llll}
\hline & NAION (n=23) & ON (n=62) & P-value \\
\hline Angiocardiopathy & $1(4.35 \%)$ & $0(0 \%)$ & $0.271 \|$ \\
Attack of eye & & & $0.009 \|$ \\
Unilateral & $23(100 \%)$ & $44(70.97 \%)$ & \\
Bilateral & $0(0 \%)$ & $18(29.03 \%)$ & \\
\hline
\end{tabular}

NAION: nonarteritic anterior ischemic optic neuropathy

ON: optic neuritis

*: statistics by the number of cases

$\dagger$ : person chi-square test

$\$$ : Independent Sample $\mathrm{T}$ test

$\S$ : Fisher's exact test

$\|$ : Correction for continuity

\subsection{Manifestations Between Nonarteritic Anterior Ischemic Optic Neuropathy and Optic Neuritis Patients}

Ocular pain $(32.5 \%$ vs. $4.35 \%, \mathrm{p}=0.007)$ and pain on movement of the eyes $(23.75 \% \mathrm{vs.} 0 \%, \mathrm{p}=0.022)$ were more common in ON patients than those in NAION patients. Vision loss in ON patients $(2.00$ vs. $1.30, \mathrm{P}=0.042)$ was significantly worse than that in NAION patients. On the contrary, optic disc edema (100\% vs. $62.5 \%, \mathrm{p}<0.001)$, peripapillary hemorrhage $(47.83 \%$ vs. $10 \%$, $\mathrm{p}<0.001)$ and peripapillary hard exudates $(17.39 \%$ vs. $1.25 \%, \mathrm{p}=0.009)$ were more common in NAION patients in comparison to ON patients (Table 2).

Table 2. Manifestations between between NAION and ON patients.

\begin{tabular}{|c|c|c|c|}
\hline & NAION $(n=23)$ & ON (n=80) & P-value \\
\hline \multicolumn{4}{|l|}{ Log MAR } \\
\hline Median & 1.30 & 2.00 & $0.042 \dagger$ \\
\hline IQR & $0.20-2.30$ & $0.85-2.53$ & \\
\hline Ocular pain & $1(4.35 \%)$ & $26(32.5 \%)$ & $0.007 \ddagger$ \\
\hline Pain on movement of the eyes & $0(0 \%)$ & $19(23.75 \%)$ & $0.022 \S$ \\
\hline Optic disc color & & & $0.265 \ddagger$ \\
\hline Light red & $17(73.91 \%)$ & $58(72.5 \%)$ & \\
\hline Red tide & $1(4.35 \%)$ & $12(15 \%)$ & \\
\hline Partial or entire pallor & $5(21.74 \%)$ & $10(12.5 \%)$ & \\
\hline Optic disc edema & $23(100 \%)$ & $50(62.5 \%)$ & $<0.001 \ddagger$ \\
\hline Retinal soft exudates & $4(17.39 \%)$ & $6(7.5 \%)$ & $0.311 \S$ \\
\hline Retinal hard exudates & $4(17.39 \%)$ & $1(1.25 \%)$ & $0.009 \S$ \\
\hline Peripapillary hemorrhage & $11(47.83 \%)$ & $8(10 \%)$ & $<0.001 \S$ \\
\hline
\end{tabular}

NAION: nonarteritic anterior ischemic optic neuropathy

ON: optic neuritis

*: statistics by the number of eyes

$\dagger$ : Mann-Whitney U test

†: person chi-square test

$\S$ : Correction for continuity

\subsection{Auxiliary Examinations Between Nonarteritic Anterior Ischemic Optic Neuropathy and Optic Neuritis Patients}

There was no difference regarding P100 amplitude $(p=0.709)$ and P100 latency $(p=0.797)$ in PVEP (Table 3$)$. The rates of partial or whole papillary hypofluorescent in early phase $(65.21 \%$ vs. $22.86 \%$, $<<0.001)$, peripapillary choroidal filling delay $(73.91 \%$ vs. $14.29 \%, \mathrm{p}<0.001)$ and partial papillary hyperfluorescent in later phase $(52.17 \%$ vs. $25.71 \%$, $\mathrm{p}=0.019$ ) were higher in NAION patients than those in ON

patients during fluorescein angiography. The rates of normal fluorescence in early phase $(13.04 \%$ vs. $60 \%, \mathrm{p}<0.001)$ and normal fluorescence in later phase $(0 \%$ vs. $20 \%, \mathrm{p}=0.046)$ in NAION patients were lower than ON patients (Table 4). No significant was observed among the patients in the rates of sector or quadrant field defect and central scotoma. The blood routine examination revealed the PLT was higher in ON patients than that in NAION patients.

Table 3. Visual Evoked Potentials (VEP) between between NAION and ON patients.

\begin{tabular}{llll}
\hline & NAION $(\mathbf{n}=\mathbf{1 6})$ & ON (n=56) & P-value \\
\hline P-VEP p100 amplitude & & 0 & $0.709 \dagger$ \\
Median & 0 & $0.00-3.61$ & \\
IQR & $0.00-4.41$ & & \\
P-VEP p100 latency & & & \\
\hline
\end{tabular}




\begin{tabular}{llll}
\hline & NAION $(\mathbf{n}=\mathbf{1 6})$ & ON $(\mathbf{n}=\mathbf{5 6})$ & P-value \\
\hline Median & 200 & 200 & $0.797 \dagger$ \\
IQR & $117.75-200.00$ & $131.10-200.00$ & \\
\hline
\end{tabular}

NAION: nonarteritic anterior ischemic optic neuropathy

ON: optic neuritis

*: statistics by the number of eyes ( In cases of severe ON or NAION with inability to respond to VEP stimulation or visual testing, values of $200 \mathrm{~ms}$ and $0 \mu \mathrm{m}$ were given for latency and amplitude.)

$\dagger$ : Mann-Whitney U test

Table 4. Fluorescein angiography between between NAION and ON patients.

\begin{tabular}{llll}
\hline & NAION (n=24) & ON (n=70) & P-value \\
\hline Peripapillary choroidal filling delay & $17(73.91 \%)$ & $10(14.29 \%)$ & $<0.001 \dagger$ \\
In early phase & & & $<2(60 \%)$ \\
Normal papillary fluorescence & $3(13.04 \%)$ & $7(10 \%)$ & $0.001 \dagger$ \\
Diffuse papillary hyperfluorescent & $0(0 \%)$ & $7(10 \%)$ & $0.262 \ddagger$ \\
Partial papillary hyperfluorescent & $7(30.43 \%)$ & $16(22.86 \%)$ & $0.041 \dagger$ \\
Partial or whole papillary hypofluorescent & $15(65.21 \%)$ & & $<0.001 \dagger$ \\
In later phase & & $14(20 \%)$ & $0.046 \dagger$ \\
Normal papillary fluorescence & $0(0 \%)$ & $34(48.57 \%)$ & $0.951 \dagger$ \\
Diffuse papillary hyperfluorescent & $11(47.83 \%)$ & $18(25.71 \%)$ & $0.019 \dagger$ \\
Partial papillary hyperfluorescent & $12(52.17 \%)$ & $8(11.43 \%)$ & $<0.001 \dagger$ \\
Partial or whole papillary hypofluorescent & $12(52.17 \%)$ & \\
\hline
\end{tabular}

NAION: nonarteritic anterior ischemic optic neuropathy

ON: optic neuritis

*: statistics by the number of eyes

$\dagger$ : person chi-square test

\$: Correction for continuity

\section{Discussion}

The main objective of this report was to show that there are several different clinical characteristics between NAION and ON. Pain on movement of the eyes and simultaneous bilateral involvement can only happen in ON rather than in NAION, which are crucial for the differential diagnosis of these two diseases. Furthermore, ON patients are younger in comparison to NAION patients. The rates of hypertension are lower in $\mathrm{ON}$ patients than in NAION patients. Ocular pain is more common in ON patients Vision loss is significantly more severe in $\mathrm{ON}$ patients than that in NAION patients. Optic disc edema, peripapillary hemorrhage and peripapillary hard exudates appear as a usual sign in NAION other than in ON. During fluorescein angiography, delayed filling in early phase, peripapillary choroidal filling delay and partial papillary hyperfluorescent in later phase are more common in NAION patients.

As our reports have shown, the average age of NAION patients was higher than in ON patients and hypertension was also more common in NAION patients. Moreover, massive studies have also demonstrated that the occurrence of NAION is not rare in patients younger than 50 years old $[10,11]$. Due to the fact that the eye is part of the entire body, many systemic diseases can play an important role in the development of several ophthalmic diseases. Thus, Hypertension, hyperlipemia, angiocardiopathy and diabetes are systemic risk factors of NAION [12]. It is prompted that systemic diseases are an important factor for the differential diagnosis between these two diseases. But in our study, this trend was observed as insignificant, even thought diabetes mellitus, hyperlipemia and angiocardiopathy were more common in NAION patients. These differences might have been statistically significant if more patients had been enrolled in the study.

In this present study, the rates of simultaneous bilateral involvement was $0 \%$ in NAION, and $29.03 \%$ in ON. which is in accordance with the Chinese NAION expert consensus, who considers the simultaneous bilateral involvement in NAION patient to be extremely rare [9]. These findings are different from Western countries, where bilateral $\mathrm{ON}$ is considered rare $(6.82 \%$ in optic neuritis) $[5,13]$.

Ocular pain and pain on movement of the eyes are crucial differentiating diagnostic features between these two diseases. Studies from Western countries demonstrate that ocular pain is an important clue to differentiate between these two diseases. In America, about $90 \%$ of ON patients suffer from ocular pain [14]. However, in Asian countries, the proportion of pain in ON patients is very low, only $40 \%$ in China and 56\% in Japan $[5,6]$. In addition, numerous studies have commented an $8-12 \%$ rates of NAION associated with ocular pain in Western countries [7]. According to the Asian populations, our study shows the rates of $\mathrm{ON}$ associated with ocular pain is $32.5 \%$ and NAION is $4.35 \%$, which shows a statistical significance in our case. Furthermore, the rates of $\mathrm{ON}$ associated with pain on movement of the eyes is $23.75 \%$ and NAION is $0 \%$. Therefore, when the disease presents without ocular pain and pain on movement of the eyes, it will be difficult to diagnose it properly, but if the patient has ocular pain or pain on movement of the eyes the probable diagnosis will be optic neuritis, where pain on movement of the eyes is more relevant than ocular pain. 
Optic disc edema and peripapillary hemorrhage were an important clinical characteristic of NAION in our study. In comparison to $\mathrm{ON}$ patients, optic disc edema and peripapillary hemorrhage were more common in NAION patients, providing a statistical difference between the two patients. This observation is consistent with other foreign researches. Moreover, the peripapillary hard exudates were also one of the most important differentiating diagnostic features between two diseases in our study.

Fluorescein angiography can provide valuable information about the differential diagnosis between NAION and ON.
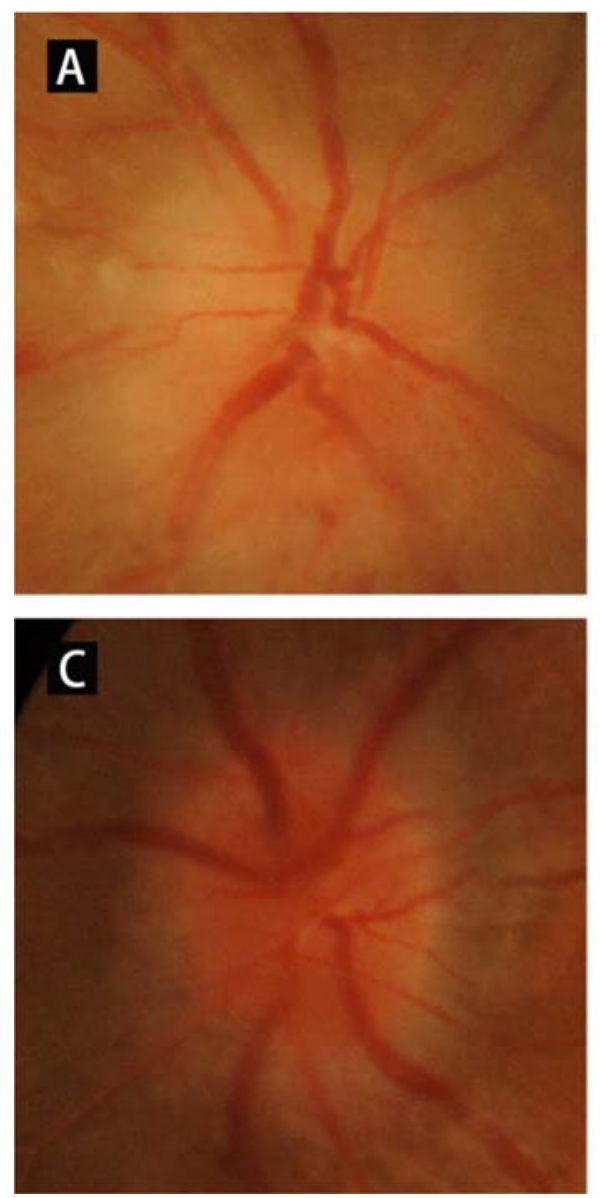

Previous studies have indicated that the optic disc and partial choroidal filling delay lead to hypofluorescent in the early phase because of the vascular insufficiency of posterior ciliary artery, and fluorescein leakage generates hyperfluorescent in later phase due to venous backflow obstruction in NAION (Figure 1) [15-17]. The typical visual field of NAION is a sector or quadrant field defect continuous with the blind spot and bypasses the fixation. (Figure 2) Whereas the visual field in $\mathrm{ON}$ is various, which mainly displays a central scotoma (Figure 2) [1, 18-19]. But no statistical difference was found between the two groups in our study.
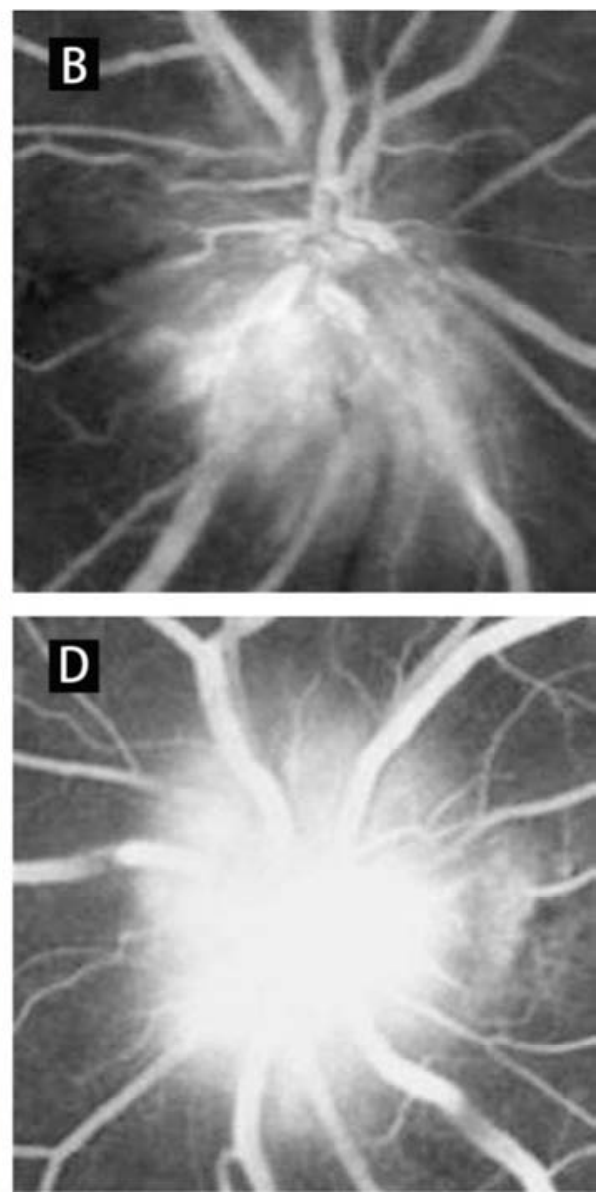

Figure 1. Comparison of the fundus photography and Fluorescein angiography: NAION with partial optic disc edema (A), and fluorescein leakage generates hyperfluorescent in later phase (B). ON with diffuse optic disc edema $(C)$ and fluorescein leakage generates hyperfluorescent in later phase (D).
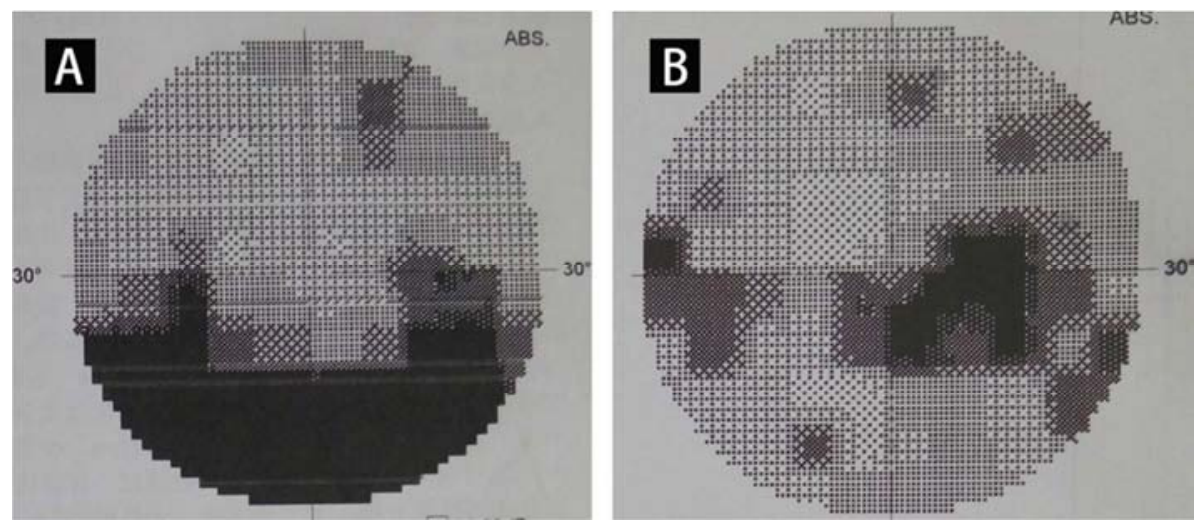

Figure 2. Comparison of the visual field: NAION showed a sector field defect continuous with the blind spot and bypasses the fixation (A). Whereas the visual field in ON mainly displays a central scotoma and enlargement of physiologic blind spot. (B). 
A p100 latency delay indicates myelin defect and a decrease in P100 amplitude means axon necrosis [20-21]. In this study, no statistical difference in P100 amplitude and P100 latency between ON patients and NAION patients. In contrast, previous researches showed P100 amplitude decreased significantly in NAION patients while P100 latency delay was more significant in ON patients of Western countries [22-24].
(Figure 3) It is probably that visual acuity loss is often severe in the early stage of $\mathrm{ON}$ in Chinese patients which indicates axon necrosis. In addition, due to the limited conditions and the small sample size of our study, sampling error is increased. For now, it is still hard for PVEP to become the key role of differential diagnosis between the two conditions in a Chinese population.
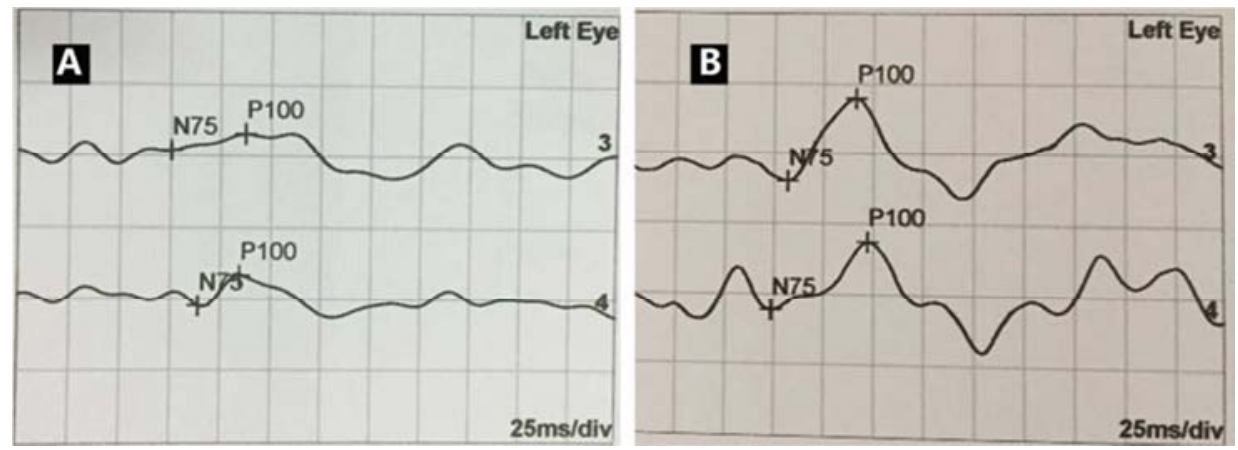

Figure 3. Comparison of the Visual Evoked Potentials: P100 amplitude decreased significantly in NAION (A) while P100 latency delay was more significant in $O N(B)$.

Recently aquaporin-4 antibody (AQP4-Ab) and myelin oligodendrocyte glycoprotein antibody (MOG-Ab) have implications for the diagnosis of neuromyelitis optica spectrum disorders (NMOSD), unfortunately, the indicators has not been included in our retrospective study. Future research is possible to observe the significance of AQP4-Ab and $\mathrm{MOG}-\mathrm{Ab}$ in the differentiation of both diseases. In addition, due to the limited conditions and the small sample size of our study, the generalization to the Chinese population is still ambitious in our study.

\section{Conclusion}

The clinical features of patients with NAION and patients with ON reveal a surprising overlap and they still have some characteristic clinical features. Pain on movement of the eyes and simultaneous bilateral involvement was observed to happen in ON rather than in NAION. Even so, in most cases, clinicians still need to associate age, gender, signs and symptoms, and auxiliary examinations in order to differentiate $\mathrm{ON}$ and NAION from each other successfully.

\section{Conflict of Interest}

None declared.

\section{References}

[1] Rizzo JF, 3rd, Lessell S. Optic neuritis and ischemic optic neuropathy. Overlapping clinical profiles [J]. Arch Ophthalmol. 1991; 109(12): 1668-1672.

[2] Wilhelm H, Schabet M. The Diagnosis and Treatment of Optic Neuritis [J]. Dtsch Arztebl Int. 2015; 112(37): 616-625; quiz 626
[3] Jianu DC, Jianu SN, Petrica L, et al. Clinical and color Doppler imaging features of one patient with occult giant cell arteritis presenting arteritic anterior ischemic optic neuropathy $[\mathrm{J}]$. Rom J Morphol Embryol. 2016, 57(2): 579-583.

[4] Nagia L, Huisingh C, Johnstone J, et al. Peripapillary Pachychoroid in Nonarteritic Anterior Ischemic Optic Neuropathy [J]. Invest Ophthalmol Vis Sci. 2016; 57(11): $4679-4685$.

[5] Du Y, Li K, Yang J, et al. Disc swelling and mild initial visual acuity loss predict a better short-term visual acuity outcome in bilateral acute optic neuritis [J]. J Clin Neurosci. 2012; 19(10): 1380-1382.

[6] Du Y, Yang J, Li JJ, et al. Unilateral optic neuritis in a Chinese population in three centers [J]. J Clin Neurosci. 2011; 18(7): 902-904.

[7] Swartz NG, Beck RW, Savino PJ, et al. Pain in anterior ischemic optic neuropathy [J]. J Neuroophthalmol. 1995, 15(1): 9-10.

[8] Neuro-ophthalmology Group of Chinese Ophthalmological Society. Diagnosis and treatment of optic neuritis expert consensus (2014). Zhonghua Yan Ke Za Zhi. 2014; (6): 459-463. (Chinese).

[9] Neuro-ophthalmology Group of Chinese Ophthalmological Society. Diagnosis and treatment of nonarteritic anterior ischemic optic neuropathy expert consensus (2015). Zhonghua Yan Ke Za Zhi. 2015; 51(5): 323-326. (Chinese).

[10] Hayreh SS. Ischaemic optic neuropathy [J]. Indian J Ophthalmol. 2000; 48(3): 171-194.

[11] Hayreh SS, Jonas JB, Zimmerman MB. Nonarteritic anterior ischemic optic neuropathy and tobacco smoking [J]. Ophthalmology. 2007; 114(4): 804-809.

[12] Kerr NM, Chew SS, Danesh-Meyer HV. Non-arteritic anterior ischaemic optic neuropathy: a review and update [J]. J Clin Neurosci. 2009; 16(8): 994-1000. 
[13] de la Cruz J, Kupersmith MJ. Clinical profile of simultaneous bilateral optic neuritis in adults [J]. Br J Ophthalmol. 2006; 90(5): 551-554.

[14] Beck RW, Cleary PA, Anderson MM, Jr., et al. A randomized, controlled trial of corticosteroids in the treatment of acute optic neuritis. The Optic Neuritis Study Group [J]. N Engl J Med. 1992; 326(9): 581-588.

[15] Shin SY, Kim DS, Ko MK. Fluorescein angiographic features of choroidal insufficiency in anterior ischemic optic neuropathy. Korean J Ophthalmol. 1999; 13(2): 100-4.

[16] Arnold AC. Fluorescein angiographic characteristics of the optic disc in ischemic and glaucomatous optic neuropathy. Curr Opin Ophthalmol. 1995; 6(2): 30-5.

[17] Kim MK, Kim US. Analysis of Fundus Photography and Fluorescein Angiography in Nonarteritic Anterior Ischemic Optic Neuropathy and Optic Neuritis. Korean J Ophthalmol. 2016; 30(4): 289-94.

[18] Hayreh SS. Ischemic optic neuropathies - where are we now. Graefes Arch Clin Exp Ophthalmol. 2013; 251(8): 1873-84.

[19] Hayreh SS, Zimmerman B. Visual field abnormalities in nonarteritic anterior ischemic optic neuropathy: their pattern and prevalence at initial examination. Arch Ophthalmol. 2005; 123(11): 1554-62.

[20] Berninger TA, Heider W. Electrophysiology and perimetry in acute retrobulbar neuritis. Doc Ophthalmol. 1989; 71(3): 293-305.

[21] Takasoh M, Mizota A, Adachi-Usami E. Comparative studies on pattern VECP between patients with ischemic optic neuropathy and optic neuritis. Acta Ophthalmol Scand. 2000; 78(4): 407-10.

[22] Janaky M, Fulop Z, Palffy A, et al. Electrophysiological findings in patients with nonarteritic anterior ischemic optic neuropathy. Clin Neurophysiol. 2006; 117(5): 1158-66.

[23] Atilla H, Tekeli O, Ornek K, et al. Pattern electroretinography and visual evoked potentials in optic nerve diseases. J Clin Neurosci. 2006; 13(1): 55-9.

[24] Mukartihal G, Radhakrishnan S, Ramasubba RM, et al. Statistical analysis of visual evoked potentials in optic neuritis and ischemic optic neuropathy subjects. Conf Proc IEEE Eng Med Biol Soc. 2005; 2: 1193-5. 